Г. А. Кононенко ${ }^{1, *}$, А. Г. Артюх ${ }^{1}$, А. Н. Воронцов ${ }^{1,2}$, Д. А. Кислуха ${ }^{1,3}$, С. А. Клыгин ${ }^{1}$, В. Е. Ковтун ${ }^{3}$, В. В. Осташко ${ }^{2}$, Ю. Н. Павленко ${ }^{2}$, Ю. М. Середа ${ }^{1,2}$, Б. Эрдэмчимэг ${ }^{1,4}$

\title{
ДЕТЕКТИРУЮЩАЯ СИСТЕМА ФРАГМЕНТ-СЕПАРАТОРА КОМБАС
}

Направлено в журнал «Приборы и техника эксперимента»

\footnotetext{
${ }^{1}$ Объединенный институт ядерных исследований, Дубна

${ }^{2}$ Институт ядерных исследований НАН Украины, Киев

${ }^{3}$ Харьковский национальный университет им. В. Н. Каразина, Харьков, Украина

${ }^{4}$ Монгольский государственный университет, Центр ядерных исследований, Улан-Батор

*E-mail: Kononenko_gennad@mail.ru
} 
Кононенко Г. А. и др.

Детектирующая система фрагмент-сепаратора КОМБАС

Представлены результаты разработки и тестирования многодетекторного телескопа $\left(\Delta E_{1}, \Delta E_{2}, E\right)$. Телескоп представляет собой компактную телескопическую комбинацию из двух 32-стриповых кремниевых $\Delta E$-детекторов и сцинтилляционных $\mathrm{CsI} / \mathrm{Tl} E$-детекторов высокой гранулярности (девять модулей) с возможностью получать из $\mathrm{Si}$-детектора сигналы для временны́х измерений (TOF). Для обслуживания мультидетекторного модуля разработаны и изготовлены как одноканальные, так и 32-канальные зарядочувствительные предусилители для кремниевых $\Delta E$ - и сцинтилляционных CsI/Tl $E$-детекторов высокой чувствительности и эффективности. В эксперименте получена однозначная идентификация по $A$ и $Z$ продуктов ядерных реакций, полученных в реакции ${ }^{40} \mathrm{Ar}$ (35 МэВ/нуклон $)+{ }^{9} \mathrm{Be}$.

Работа выполнена в Лаборатории ядерных реакций им. Г. Н. Флерова ОИЯИ.

Kononenko G. A. et al.

P13-2014-20

Detection System of the COMBAS Fragment Separator

The results of design and testing of multi-detector telescope $\left(\Delta E_{1}, \Delta E_{2}, E\right)$ are given. The compact telescope consists of 32-strip Si $\Delta E$-detectors and CsI/Tl $E$ scintillation detectors of high granularity (nine modules) and allows one to obtain timing signal from Si detectors for TOF measurements. The single channel and 32-channel charge-sensitive preamplifiers were developed and manufactured to serve the $\mathrm{Si} \Delta E$-detectors and $\mathrm{CsI} / \mathrm{Tl}$ scintillation $E$-detectors with high efficiency and sensitivity. Unambiguous $A$ and $Z$ identification of reaction products, produced in the ${ }^{40} \mathrm{Ar}$ (35 MeV/nucleon) $+{ }^{9} \mathrm{Be}$ reaction system, is implemented.

The investigation has been performed at the Flerov Laboratory of Nuclear Reactions, JINR. 


\section{ВВЕДЕНИЕ}

Исключительное многообразие ядерных реакций с тяжелыми ионами, огромное число возможных комбинаций «ион - ядро мишени» открывают благоприятные перспективы как для получения и формирования вторичных пучков ускоренных радиоактивных ядер с аномальным отношением $N / Z$, так и для исследования структуры экзотических ядер. Реакции с тяжелыми ионами являются весьма эффективными в синтезе неизвестных изотопов, лежащих на границе или даже за границей нуклонной стабильности.

В настоящее время для получения и сепарации вторичных пучков радиоактивных ядер с определенными значениями $A$ (массовое число) и $Z$ (атомный номер) используются в основном кинематические (in-flight) сепараторы $[1,2]$. Однако получить моноизотопные пучки вторичных радиоактивных ядер достаточной интенсивности методом in-flight-сепарации невозможно без значительных потерь их интенсивности. Известно, что в реакциях передачи и фрагментации продукты ядерных реакций получаются с широкими импульсными распределениями и в различных зарядовых состояниях $(Q)$. Вследствие этого моноизотопную сепарацию продуктов ядерных реакций невозможно обеспечить только магнитной жесткостью $B \rho$, поскольку магнитная жесткость сепаратора $B \rho$ является функцией нескольких параметров анализируемых частиц, а именно:

$$
B \rho \sim P / Q=A v /(Z-n) .
$$

Здесь $B$ - магнитное поле сепаратора (Тл), а $\rho$ - радиус центральной траектории сепарируемой частицы (м), $P$ - импульс частицы, а $Q=$ $(Z-n)$ - зарядовое состояние иона, равное разности заряда ядра $Z$ и числа подхваченных $n$-электронов, $v$ - скорость транспортируемых частиц (м/с).

Следовательно, для однозначной идентификации по $A$ и $Z$ продуктов ядерных реакций, включая и их зарядовые состояния, требуется многопараметровая система их регистрации. По этой причине детектирующая система фрагмент-сепаратора КОМБАС формируется из каскада прострельных $X$ - и $Y$-координатных детекторов различной толщины, измеряющих ионизационные потери энергии $\Delta E$ частицами, детектора поглощения остаточной энергии $E_{r}$ высокой гранулярности и измерения времени пролета TOF, а именно: 


$$
\Delta E \sim(Z / v)^{2}, \quad \Delta E+E_{r} \sim\left(A / v^{2}\right) / 2 \quad \text { и } \quad \text { TOF } \sim(1 / v) .
$$

Такой вариант детектирующей системы в комбинации с магнитным анализом позволяет одновременно регистрировать весь спектр транспортируемых частиц: легкие длиннопробежные продукты (занята вся толщина детекторов) и короткопробежные сильноионизирующие частицы с большими атомными номерами (используется только несколько передних $\Delta E$-детекторов). Анализ показывает, что набор параметров $B \rho, \Delta E, E_{r}$ и ТOF является достаточным для однозначной идентификации по $A$ и $Z$ продуктов ядерных реакций, включая и их зарядовые состояния.

Целью данной работы является создание серии детектирующих модулей $\left(\Delta E_{1}, \Delta E_{2}, E_{r}\right)$, которые могли бы обеспечить достаточный телесный угол регистрации, высокое угловое разрешение и однозначную идентификацию всего спектра продуктов ядерных реакций, транспортируемых в выходную фокальную плоскость сепаратора КОМБАС. Каждый модуль представляет собой компактную телескопическую комбинацию из 32-стриповых $(X, Y)$ кремниевых $\Delta E$-детекторов и сцинтилляционных $\mathrm{CsI} / \mathrm{Tl} E$-детекторов высокой гранулярности (девять детекторов) с возможностью получать из Siдетекторов сигналы для TOF-измерений. Для обслуживания гибридного ( $\mathrm{Si}$ и CsI/Tl) телескопа было необходимо разработать и создать многоканальные (32-канальные) зарядочувствительные предусилители высокой чувствительности и эффективности.

\section{1. КОНСТРУКЦИЯ ТЕЛЕСКОПА}

Телескопический модуль монтируется в вакууме приемной камеры фрагмент-сепаратора КОМБАС [2]. Детекторная часть телескопа состоит из двух кремниевых прострельных $X$ - и $Y$-координатных стриповых $\Delta E$ детекторов большой площади $(66 \times 66$ мм каждый) и расположенной за ними гранулированной стенки из девяти (матрицы $3 \times 3$ ) сцинтилляционных $\Delta E$ детекторов полного поглощения энергии. Стриповые детекторы предназначены как для спектрометрических и угловых измерений потока частиц, так и для определения профиля их распределения в позиции ахроматического выходного фокуса фрагмент-сепаратора. Сцинтилляционные детекторы полного поглощения необходимы для измерения энергии длиннопробежных продуктов ядерных реакций, которые простреливают оба стриповых детектора. Фотографии телескопа приведены на рис. 1.

На рис. 1, $a$ в качестве первого по пучку установлен тонкий (380 мкм) стриповый $\mathrm{Si}$-детектор, ориентированный так, чтобы стрипы располагались вертикально ( $X$-координатный детектор). Вслед за ним на расстоянии 10 мм 

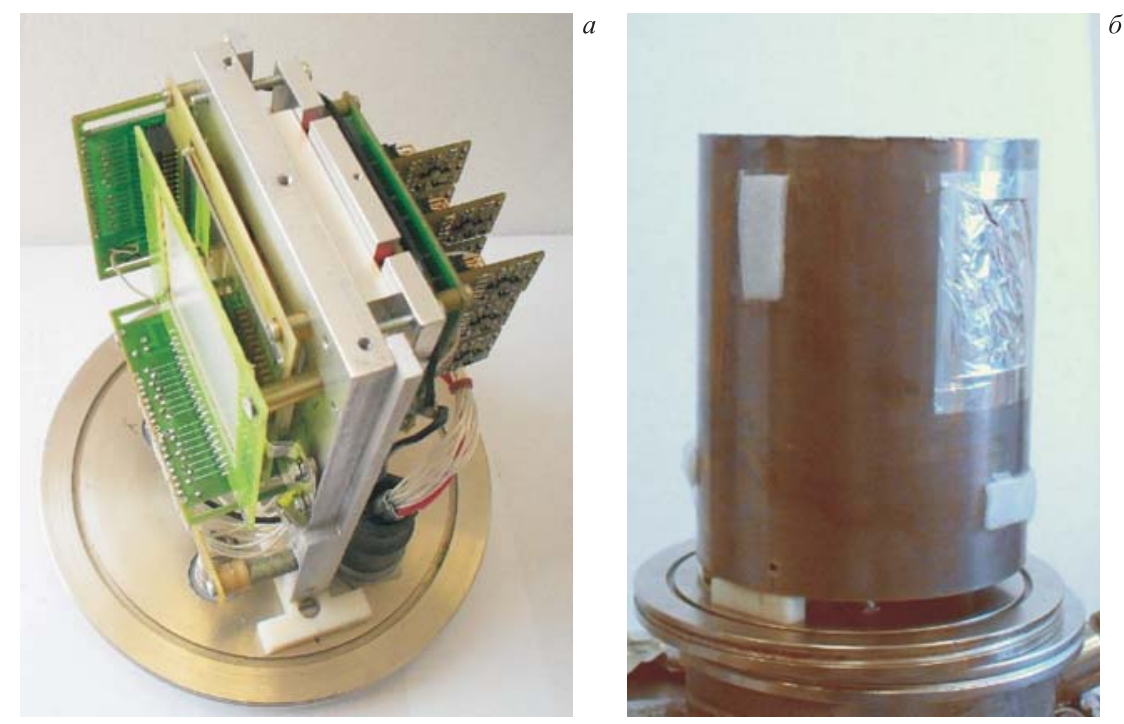

Рис. 1. Телескоп детекторов с предусилителями, смонтированный на вакуумной заглушке диаметром 180 мм: $a-$ со снятым кожухом; $\sigma-$ в полной сборке. Размеры кожуха: диаметр 135 мм, высота 170 мм

размещен стриповый Si-детектор толщиной 1 мм. Он установлен с ортогональным расположением стрипов по отношению к первому детектору ( $Y$ координатный детектор). Фотография одного из стриповых детекторов приведена на рис. 2. Размеры стриповых детекторов по плоскости $66 \times 66$ мм. Они содержат по 32 стрипа длиной 60 мм и шириной 1,9 мм каждый. Шаг стрипов 2 мм.

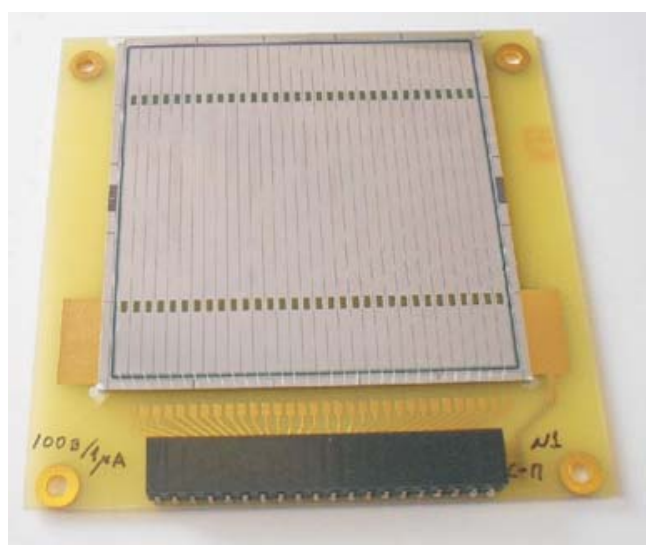

Рис. 2. Стриповый детектор 
В телескопе стриповые детекторы устанавливаются в разъемы на текстолитовых платах, которые закреплены на прямоугольной рамке с окном. Рамка крепится к двум стойкам. Стойки через изолирующую прокладку фиксируются на диске вакуумной заглушки (рис. 1). Детекторы изготовлены группой ФТИ-НИИМВ (Физико-технический институт им. А.Ф.Иоффе и Научноисследовательский институт материаловедения).

Вслед за $Y$-стриповым детектором на расстоянии 10 мм размещена матрица из девяти сцинтилляционных детекторов полного поглощения энергии частиц. Каждый из них состоит из сцинтилляционного кристалла CsI/Tl в сочетании с кремниевым $p$ - $i$ - $n$-фотодиодом (PD) (рис. 3 ).
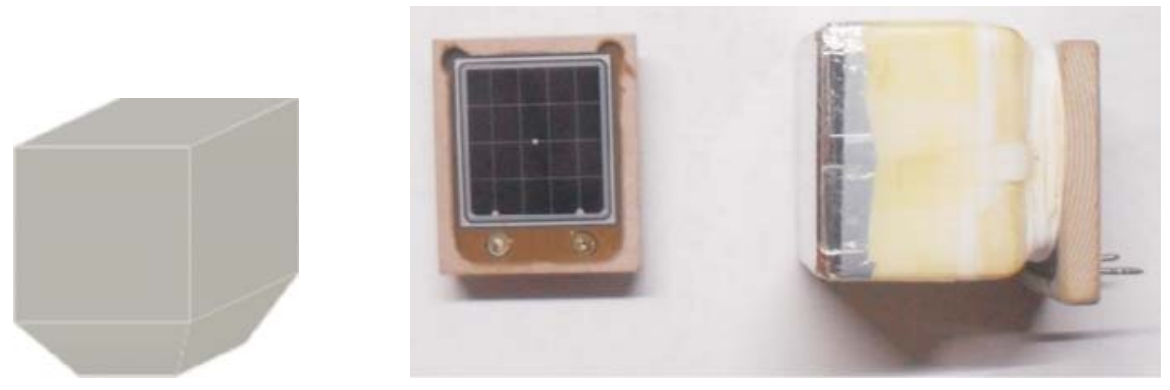

Рис. 3. Внешний вид кристалла сцинтиллятора CsI/Tl, кремниевого $p$-i-n-фотодиода и собранного детектора

Для сцинтилляционных детекторов нами были использованы бескорпусные $p$ - $i$-n-диоды с площадью поверхности $S=10,6 \times 11,6$ мм и толщиной чувствительного слоя $d_{0}=300$ мкм. Емкость фотодиодов составляет $C_{D} \approx(1$ пФ/см· $\left.S) / d_{0}\right) \approx 40$ пФ. Отдельно изготавливался керамический корпус, в котором устанавливались контакты. После установки и распайки в нем фотодиода он заливался эпоксидной смолой.

Фрезеровкой сцинтилляционному кристаллу CSI/Tl придавалась сложная форма, состоящая из прямоугольного параллелепипеда с размерами $18 \times 18 \times$ 13,5 мм и пирамидальной части. Высота пирамидальной части составляет 3,6 мм. Верхнее основание $18 \times 18$ мм, а нижнее $-11 \times 11$ мм, совпадающее с размерами окна фотодиода. Полный объем сцинтиллятора составляет таким образом 5,1 см ${ }^{3}$. Боковые стенки кристалла обрабатываются наждачной бумагой (№ 400) до получения матовой поверхности. И в дальнейшем покрываются диффузным материалом, не пропускающим свет. В данном случае используются 2-3 слоя тефлоновой ленты толщиной 0,1 мм.

Передняя поверхность, обращенная к пучку, обрабатывается более мелкой (№ 1500) наждачной бумагой с уайт-спиритом и покрывается майларовой фольгой толщиной 2-5 мкм. Применение растворителя обусловлено тем, что 
он позволяет избежать грубых повреждений поверхности кристалла и сделать ее рельеф более однородным. Поверхность кристалла, прилегающая к фотодиоду, полируется до блеска. В дальнейшем сцинтиллятор с помощью оптического клея крепится к фотодиоду.

Девять кристаллов с приклеенными фотодиодами объединяются в матрицу $3 \times 3$ и закрепляются в рамке-держателе, которая размещается вблизи прострельных стриповых Si-дететкторов. Фотографии CsI/Tl-кристалла сцинтиллятора, кремниевого фотодиода, установленного в керамическую оправку, и собранного детектора приведены на рис. 3.

На рис. 4 представлена сборка из девяти сцинтилляционных CsI/Tl PDдетекторов. Детекторы устанавливаются в ячейки с разъемами на общей печатной плате. На этой же плате в разъемах устанавливаются зарядочувствительные предусилители. Печатная плата с детекторами и предусилителями крепится к несущей рамке, в которой выбрано окно под кристаллы. С лицевой части несущей рамки в пазах установлены четыре обжимающие планки с уплотнителями из вакуумной резины. Они могут перемещаться по пазам рамки и жестко закрепляться на ней. С их помощью происходит механическая фиксация детекторов. Несущая рамка со сцинтилляционными детекторами крепится винтами с обратной стороны к прямоугольной рамке со стриповыми детекторами.

Сигналы с каждого стрипа $X$ - и $Y$-детекторов, а также с их общих электродов короткими кабелями заведены на свои ( $X$ или $Y$ ) вакуумные 50-штырьковые разъемы ОСРС50ТВ. Ответные части (проходные разъемы) РСГСП 50-В установлены на диске вакуумной заглушки. Напряжения смещения для каждого из детекторов подаются через два герметичных разъема СРГ-50-82фв, которые также установлены на диске. На отдельный разъем

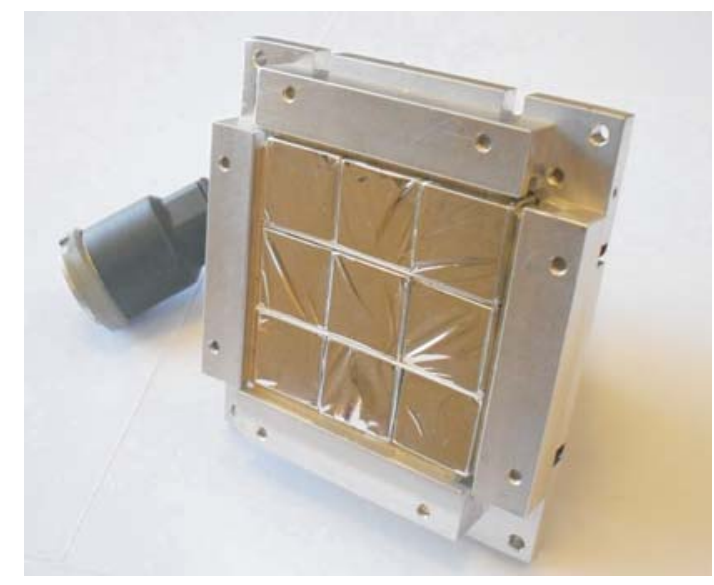

Рис. 4. Матрица $3 \times 3$ сцинтилляционных CsI/Tl PD-детекторов 


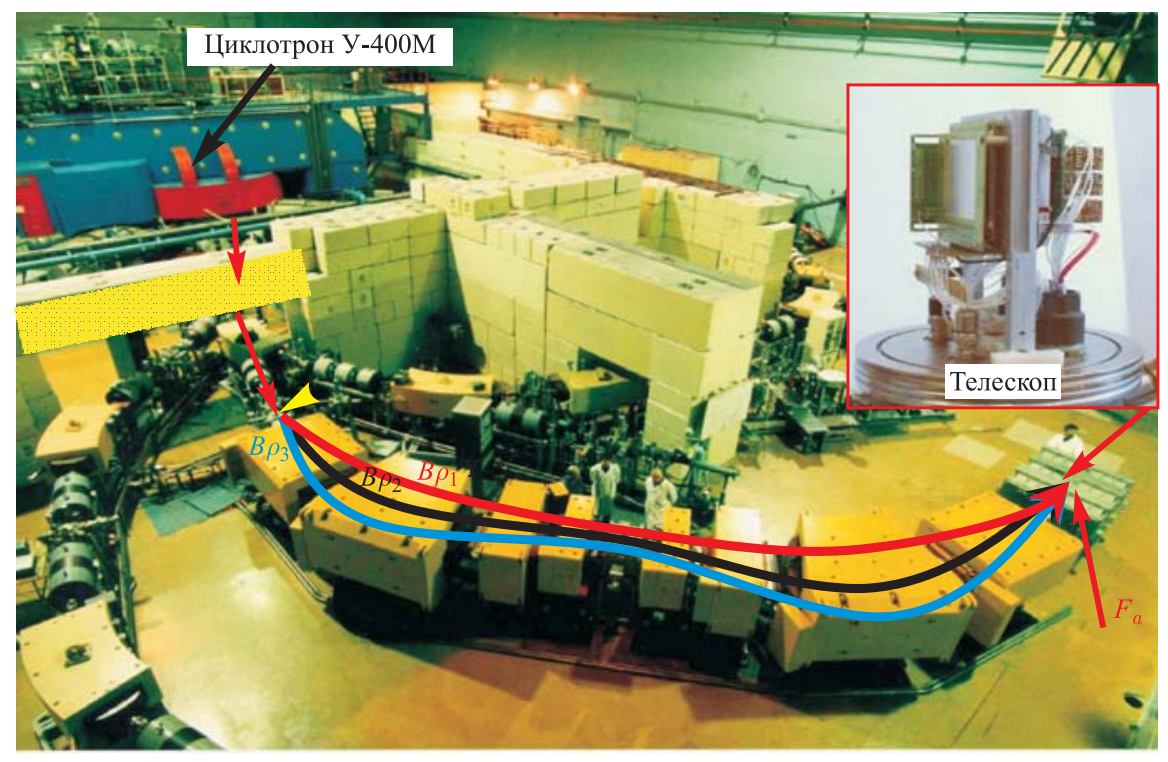

Рис. 5. Фрагмент-сепаратор КОМБАС, размещенный в экспериментальном зале циклотрона У-400М Лаборатории ядерных реакций им. Г.Н. Флерова Объединенного института ядерных исследований (Дубна)

ОСРС50ТВ выведены выходные сигналы предусилителей сцинтилляционных детекторов. На этот же разъем заведены выходы с общих электродов стриповых детекторов, напряжение смещения (+60B) CsI/Tl PD-детектров, тестовый сигнал и напряжения питания для предусилителей сцинтилляционных детекторов.

Телескоп крепится в вакууме приемной камеры на фланце, которая располагается в позиции выходного ахроматического фокуса $F_{a}$ фрагментсепаратора КОМБАС (рис. 5).

\section{2. ЗАРЯДОЧУВСТВИТЕЛЬНЫЕ ПРЕДУСИЛИТЕЛИ}

Детекторный модуль имеет 75 спектрометрических каналов регистрации, каждый из которых снабжен своим зарядочувствительным предусилителем с возможностью индивидуального считывания. Так как емкости сцинтилляционных детекторов ( $\approx 40$ пФ) и отдельных стрипов $(\approx 26$ пФ - для 380-мкм детектора и $\approx 12$ пФ - для 1-мм) можно считать небольшими, для них предусилители были выполнены по простейшей классической схеме [3,4]. Предусилитель состоит из входного каскада с каскадным включением (JFET-pnp) транзисторов [5] и составного эмиттерного повторителя на выходе. Пред- 
усилители изготовлены на элементах поверхностного монтажа в виде небольших модулей со штыревыми разъемами размерами $25 \times 11$ мм (рис. 6).

Со стороны общих электродов емкость стриповых детекторов гораздо выше: $\approx 1000$ пФ для 380-мкм и $\approx 390$ пФ для 1-мм детекторов. Поэтому для регистрации сигналов с общих электродов были изготовлены несколько зарядочувствительных предусилителей с более высокими коэффициентами усиления при разомкнутой отрицательной обратной связи. Входной каскад в них выполнен аналогично, а вместо нагрузочного резистора в коллекторе каскада включена активная нагрузка на отдельном транзисторе (генератор тока) [6] и на выходе установлен буферный элемент (рис. 7).

Во входных каскадах предусилителей для сцинтилляционных детекторов, работающих в вакууме, применены полевые транзисторы BF861А с низким рабочим током стока (5 мА). Это позволило добиться мощности потребления

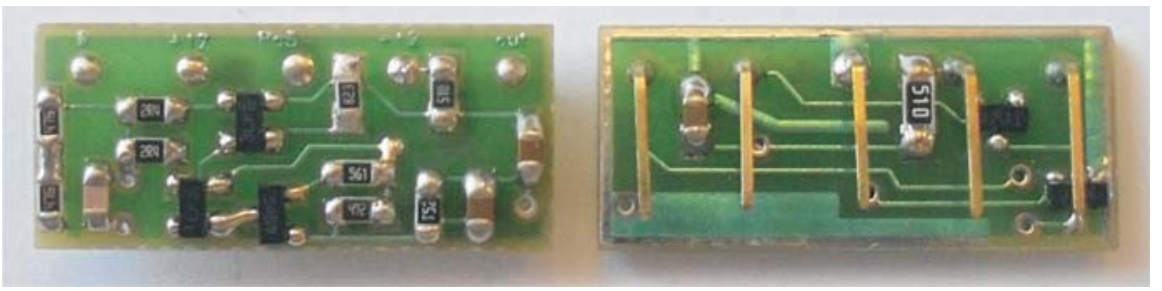

Рис. 6. Предусилитель крупным планом

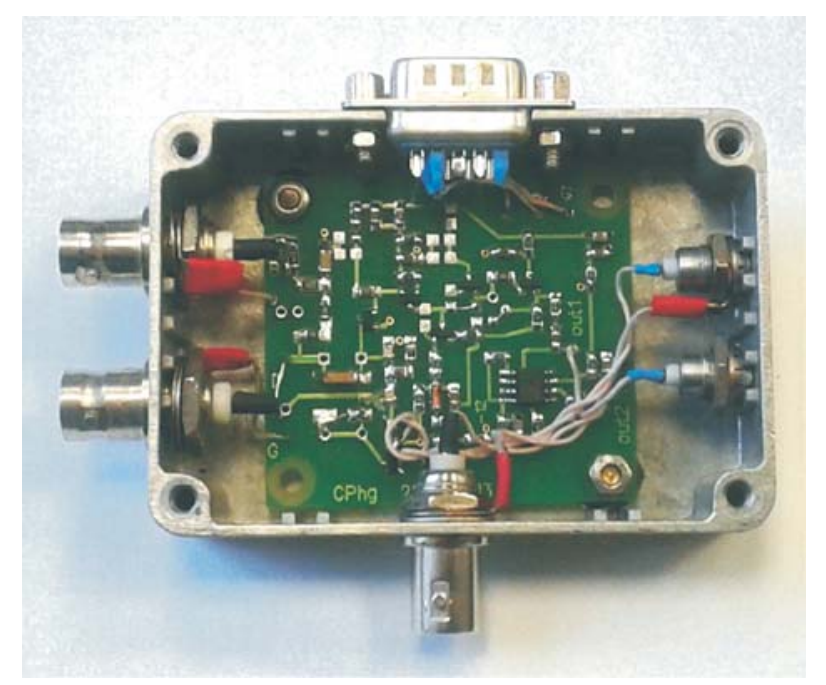

Рис. 7. Предусилитель для общих электродов стриповых детекторов 


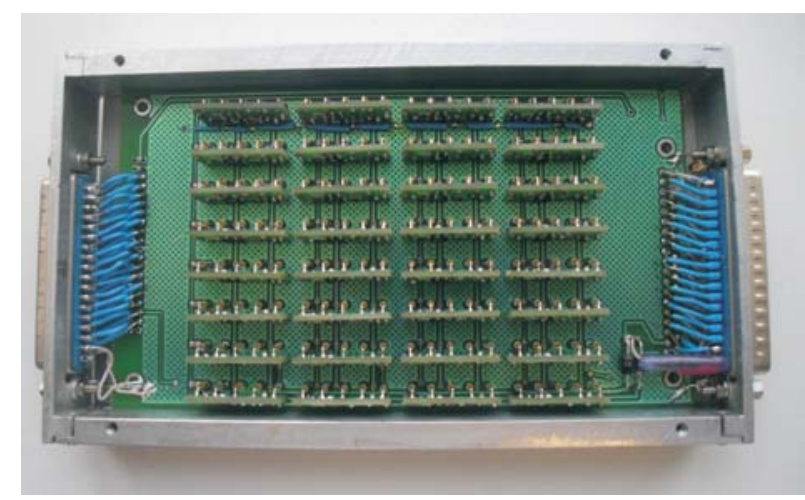

Рис. 8. Кассета с 32 предусилителями для стрипового детектора, размещаемая вне вакуумной камеры приемного устройства фрагмент-сепаратора КОМБАС

от источников питания для одного предусилителя в 100 мВт. В предусилителях для стриповых детекторов использованы транзисторы BF862.

В детекторном модуле установлены предусилители только для девяти CsI/Tl PD-детекторов (рис. 4). Предусилители для стриповых детекторов размещены в индивидуальных гнездах со штыревыми разъемами по 32 штуки в кассетах (рис.8), которые расположены вне вакуумной камеры приемного устройства фрагмент-сепаратора КОМБАС и соединяются с соответствующими разъемами детекторов короткими (20 см) кабелями. Рядом с ними расположены два предусилителя, подключаемых к общим электродам стриповых детекторов.

Тестирование предусилителей и детекторов осуществлялось на стенде, показанном на рис.9. Помимо стандартного в таких случаях спектрометрического оборудования он включал вакуумную камеру с телескопом (рис. 1)

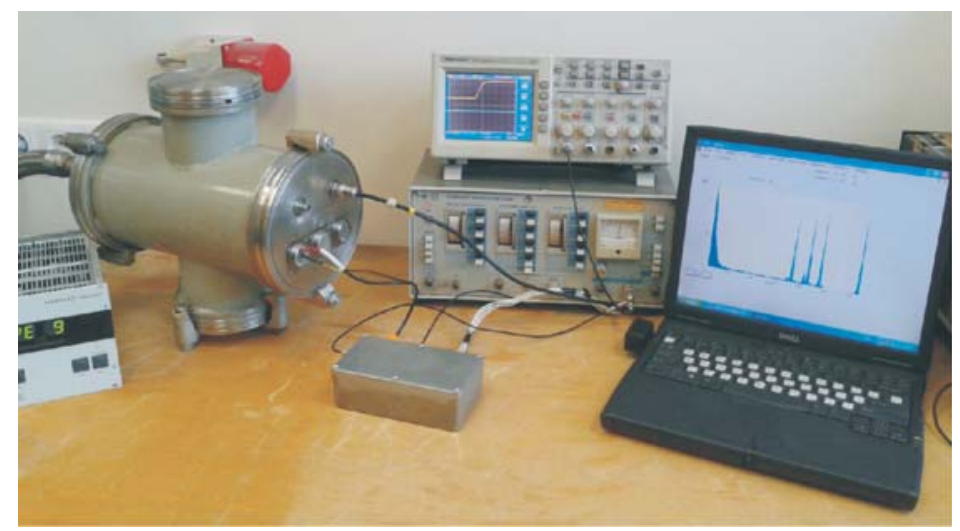

Рис. 9. Стенд для тестирования предусилителей и детекторов 
и электронным экранированным блоком, в котором через разъем устанавливался необходимый предусилитель.

Все предусилители при измерении их шумовых характеристик тестировались без подключения детектора $\left(C_{D}=0\right)$ и были настроены на коэффициент преобразования $G_{p} \approx 1,0 \mathrm{~B} / п$ Кл, что соответствует чувствительности в энергетических единицах $G_{E} \approx 44 \mathrm{MB} / \mathrm{MэВ} \mathrm{для} \mathrm{кремниевого} \mathrm{детектора.}$

Шум предусилителя, приведенный ко входу в виде эквивалентного шумового заряда ENC (Equivalent Noise Charge) в среднеквадратичных (r. m.s.) электронах, вычислялся как

$$
\mathrm{ENC}=U_{N}(\text { r.m.s. }) / G_{p} G_{\mathrm{amp}} e^{-},
$$

где $U_{N}$ (r. m. s.) - среднеквадратичное значение напряжения шума на выходе спектроскопического усилителя, $G_{\text {amp }}$ - коэффициент усиления спектрометрического усилителя, а $e^{-}$- заряд электрона.

Так как измеряемый шумовой сигнал представляет собой белый шум с гауссовым распределением и нулевым средним значением, для него стандартное отклонение $\sigma$ равно среднеквадратичному значению измеряемого параметра $U_{N}$ (r.m.s.). Поэтому с точностью 99,73\% $( \pm 3 \sigma) U_{N}$ (r.m.s.) $=U$ (peak-to-peak) : 6 [7]. Амплитудный размах $U$ (peak-to-peak) шумового напряжения измерялся цифровым осциллографом TDS 2024 на выходе спектрометрического усилителя БУИ-3К с постоянной времени формирования в 1 мкс и коэффициентом усиления $G_{\mathrm{amp}}=100$ с подключенным предусилителем и затем без него.

Для примера на рис. 10 приведены две типичные осциллограммы шумовых сигналов, полученные при исследовании одного из предусилителей. Слева - шумы на выходе усилителя БУИ-3К с подключенным предусилите-
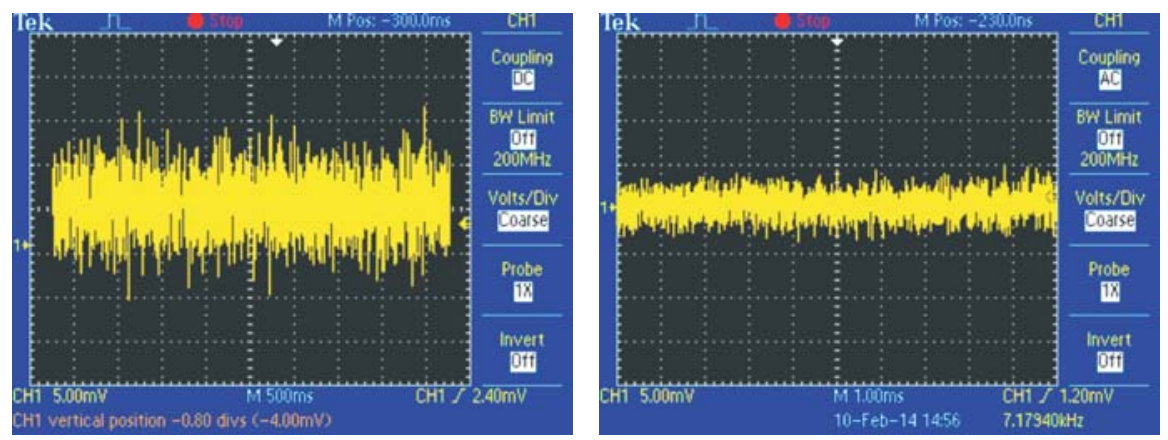

Рис. 10. Шумовые сигналы на выходе спектрометрического усилителя с подключенным к нему предусилителем (левая осциллограмма) и без предусилителя (правая осциллограмма) 
лем; справа - шумы на выходе усилителя без предусилителя. Амплитудные размахи этих сигналов составили: $U_{N 1}$ (peak-to-peak) $=23 \mathrm{мB}-$ амплитудный размах шумового сигнала левой осциллограммы и $U_{N 1}$ (peak-to-peak) $=8 \mathrm{MB}-$ амплитудный размах сигнала по правой осциллограмме.

По этим данным среднеквадратичное значение напряжения шума $U_{N}$ (r.m.s.) $\approx 3,6$ мB, а значение $\mathrm{ENC} \approx 225$ электронов r. m. s.

В энергетических единицах в пересчете для кремниевого детектора: $\mathrm{FWHM}=2,355 \cdot \varepsilon \cdot \mathrm{ENC}=2,355 \cdot 3,61 \cdot 225 \approx 1,9 \mathrm{\kappa эB.}$

Здесь $\varepsilon=3,61$ эВ - энергия, затрачиваемая на образование одной пары заряженных частиц в кремнии.

Наклон шумовой характеристики предусилителей, предназначенных для общих электродов стриповых детекторов, составляет 16 эВ/пФ.

\section{3. ХАРАКТЕРИСТИКИ ДЕТЕКТОРОВ}

Сцинтилляционные CsI/Tl-детекторы. $p$-i-n-фотодиоды для сцинтилляционных детекторов отбирались с минимально возможным темновым током (1-3 нА). До постановки диода в керамический корпус для каждого из них измерялось напряжение смещения, обеспечивающее полное обеднение $p$-n-перехода. Для этого диоды облучались с обратной стороны источником альфа-излучения ( $\left.{ }^{241} \mathrm{Am}\right)$, и при разных напряжениях смещения измерялось положение пика альфа-частиц в амплитудном спектре (рис. 11).

После установки в керамический корпус, распайки и заливки эпоксидной смолой каждый фотодиод проверялся на уровень шумов и величину темнового тока.

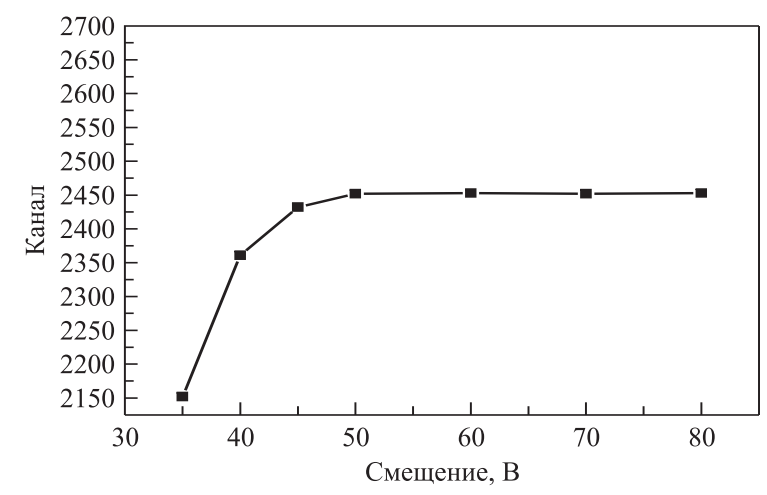

Рис. 11. Положение пика альфа-частиц ${ }^{241} \mathrm{Am}, E_{\alpha}=5,485$ МэВ, в зависимости от напряжения смещения на $p-i-n$-диоде. Диод облучался с обратной стороны 

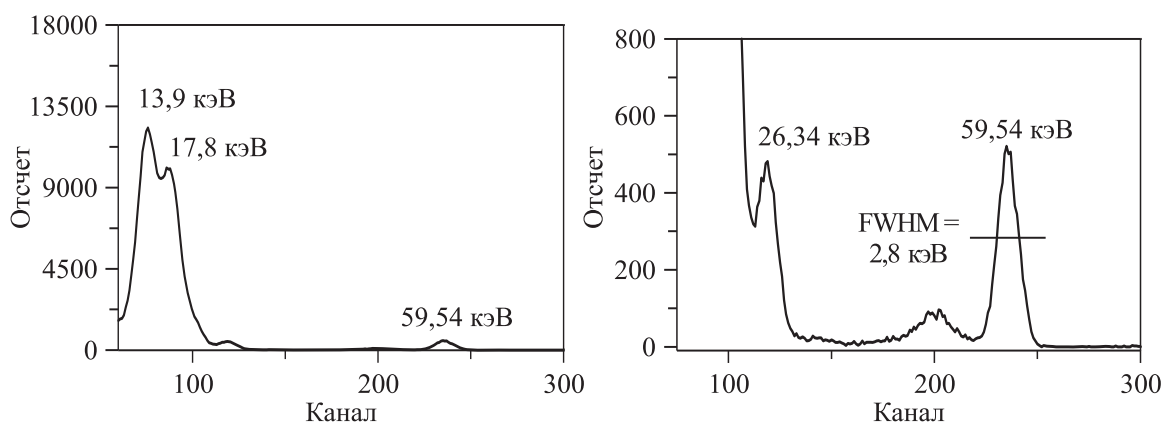

Рис. 12. Рентгеновский спектр ${ }^{241} \mathrm{Am}$, измеренный кремниевым $p$-i-n-фотодиодом $C_{D} \approx 40$ пФ, площадью $S=10,6 \times 11,6$ мкм и толщиной 300 мкм

Шумовые свойства PD оценивались по значению полуширины рентгеновского пика 59,54 кэВ в спектре ${ }^{241} \mathrm{Am}$, получаемого от исследуемого фотодиода. На рис. 12 представлен один из таких рентгеновских спектров. Данный спектр был получен для диода с темновым током в 3 нА и напряжением смещения $U_{B}=60 \mathrm{~B}$. Спектрометрический тракт состоял из рассмотренного выше предусилителя, спектрометрического усилителя БУИ-3К с коэффициентом усиления $K=100$ и постоянной времени формирования $\tau=1$ мкс. Энергетическое разрешение $\Delta E(\mathrm{FWHM}) / E$ для линии 59,54 кэВ составило $4,7 \%$.

Качество изготовленных сцинтилляторов оценивалось по получаемому энергетическому разрешению $R=\Delta E(\mathrm{FWHM}) / E$ для $\gamma$-линий полного поглощения 1332 кэВ $\left({ }^{60} \mathrm{Co}\right)$.

На рис. 13 приведен типичный $\gamma$-спектр ${ }^{60} \mathrm{Co}$, полученный для одного из сцинтилляторов CsI/Tl PD-детекторов, входящих в матрицу телескопа.

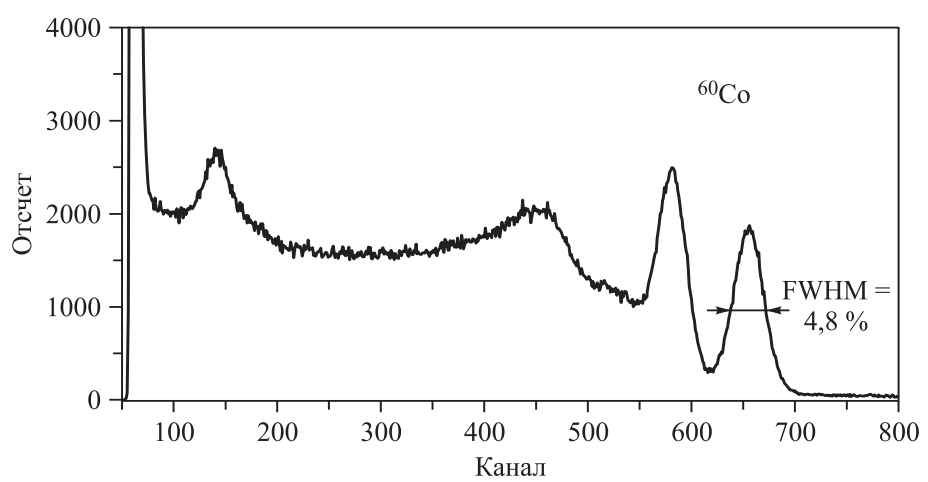

Рис. 13. Гамма-спектр ${ }^{60} \mathrm{Co}$, измеренный CsI/Tl PD-детектором с объемом кристалла $5,1 \mathrm{~cm}^{3}$ 


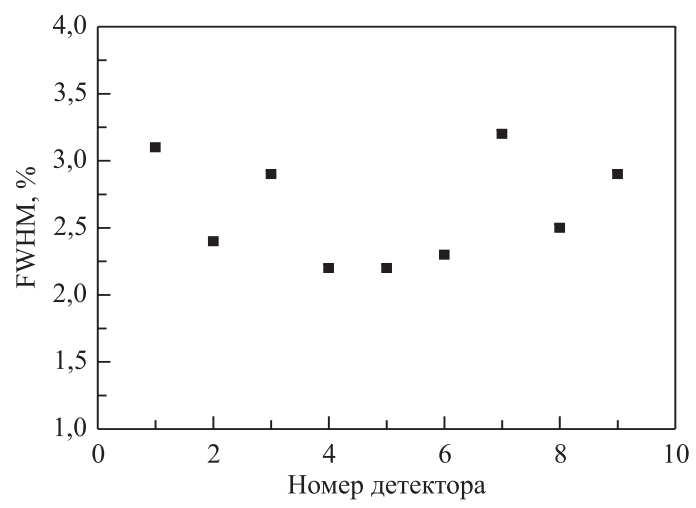

Рис. 14. Разрешение FWHM (\%) матрицы из девяти CSI/TL PD-детекторов. Источник ${ }^{226} \mathrm{Ra}$ в вакууме

На рис. 14 представлено распределение разрешений CsI/Tl PD-детекторов в матрице из девяти $(3 \times 3)$ телескопов. Разрешение определялось как полуширина (FWHM, \%) пика $\alpha$-частиц с энергией 7687 кэВ источника ${ }^{226} \mathrm{Ra}$. Детекторы облучались источником в вакууме.

Стриповые детекторы. Напряжение полного обеднения для обоих детекторов было измерено по такой же методике. Для 380-мкм стрипового детектора оно составило $U_{B}=120 \mathrm{~B}$ и $U_{B}=150 \mathrm{~B}$ - для 1-мм детектора. Для этих напряжений значения темновых токов $\left(I_{0}\right)$ для тонкого детектора составило 240 нА, а для 1-мм детектора 0,95 мкА.

Постриповое разрешение детекторов измерялось через коллимированный источник ${ }^{226} \mathrm{Ra}$ на стенде (рис.9) в вакуумном боксе. Среднее значение разрешения для обоих детекторов составило $1 \%$. На рис. 15 приведено постриповое разрешение 1-мм детектора. Как и в случае со сцинтилляционными

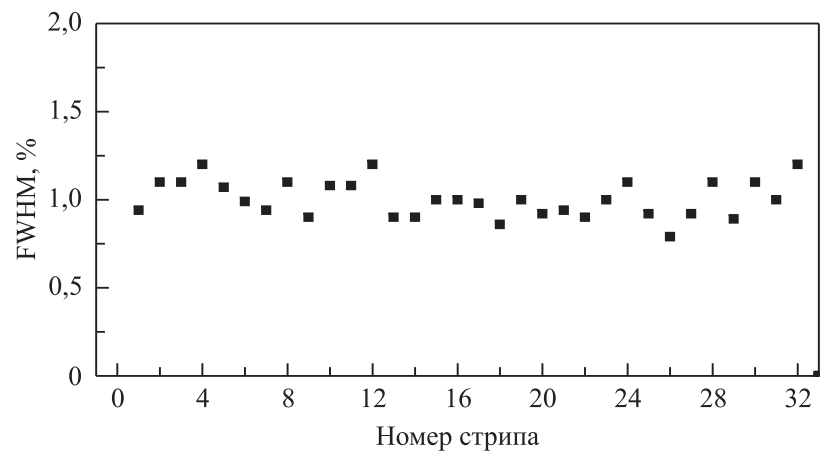

Рис. 15. Постриповое разрешение FWHM (\%) 1-мм детектора $\left(U_{B}=150 \mathrm{~B}, I_{0}=\right.$ 950 нА). Источник ${ }^{226} \mathrm{Ra} \mathrm{в} \mathrm{вакууме}$ 


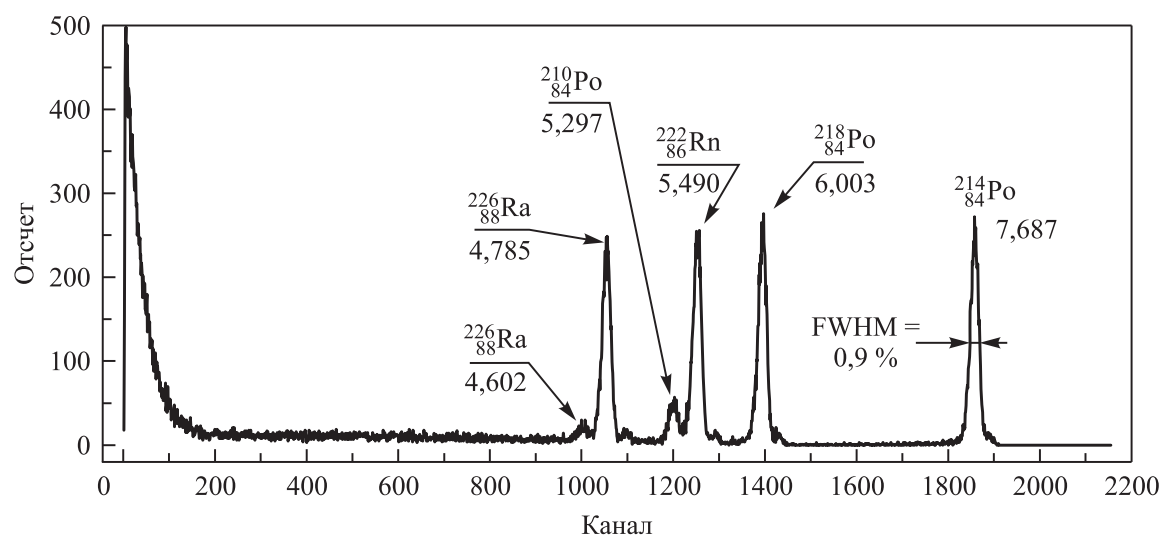

Рис. 16. Спектр альфа-частиц ${ }^{226} \mathrm{Ra}$, полученный от одного стрипа детектора толщиной 1 мм

CsI/Tl PD-детекторами, разрешение определялось по полуширине пика $\alpha$ частиц с энергией 7687 кэВ источника ${ }^{226} \mathrm{Ra}$.

На рис. 16 показан $\alpha$-спектр источника ${ }^{226} \mathrm{Ra}$, полученного от одного из стрипов 1-мм детектора при облучении в вакууме.

\section{ЗАКЛЮЧЕНИЕ}

Представлены результаты создания и тестирования многодетекторного измерительного телескопа ( $\left.\Delta E_{1}, \Delta E_{2}, E\right)$, который в состоянии обеспечить значительный телесный угол регистрации и однозначную идентификацию по $A$ и $Z$ продуктов ядерных реакций, транспортируемых в выходную фокальную плоскость фрагмент-сепаратора КОМБАС. Модуль представляет собой компактную телескопическую комбинацию из двух 32-стриповых кремниевых $\Delta E$-детекторов и сцинтилляционных $\mathrm{CsI} / \mathrm{Tl} E$-детекторов высокой гранулярности (девять модулей) с возможностью получать из Si-детектора сигналы для временны́х измерений ТОF. Для обслуживания мультидетекторного модуля были разработаны и изготовлены зарядочувствительные предусилители для кремниевых $\Delta E$ - и сцинтилляционных $\mathrm{CsI} / \mathrm{Tl} E$-детекторов высокой чувствительности и эффективности.

Качество изотопной идентификации продуктов ядерных реакций, полученных в реакции ${ }^{40} \mathrm{Ar}$ (35 МэВ/нуклон) + ${ }^{9}$ Ве с помощью фрагментсепаратора КОМБАС и рассмотренной детектирующей системы, иллюстрирует рис. 17.

Как видно из представленной матрицы на рис. 17, комбинация фрагментсепаратора КОМБАС с созданным телескопическим измерительным модулем 


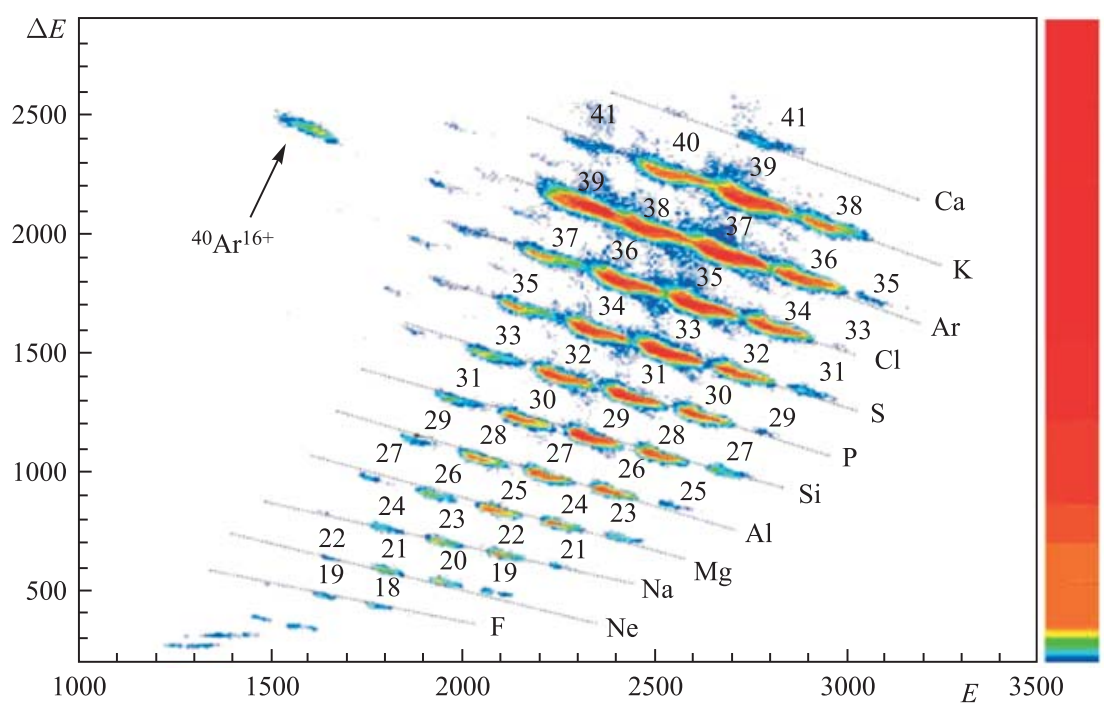

Рис. 17. Идентификационная матрица продуктов ядерных реакций с атомными номерами $4<Z<20$, полученная в реакции ${ }^{40} \mathrm{Ar}(E=35 \mathrm{MэB/A})$ с мишенью ${ }^{9} \mathrm{Be}$ $\left(15\right.$ мг/см $\left.{ }^{2}\right)$. Продукты реакций выделялись фрагмент-сепаратором КОМБАС и транспортировались в выходной фокус в позицию телескопа $\Delta E$ - $E$-детекторов

обеспечивает высокую разрешающую способность и позволяет однозначно определять значения массовых чисел $A$ нуклидов и атомных номеров $Z$ широкого спектра продуктов ядерных реакций.

Детектирующая система фрагмент-сепаратора КОМБАС, созданная из комплекса таких телескопических измерительных модулей, является чрезвычайно перспективной для измерения редких многочастичных распадов экзотических ядер. Исследования таких процессов могут быть эффективными, если детектирующая система в состоянии обеспечить регистрацию частиц в конусе максимально большого телесного угла и высокое угловое разрешение коррелированных кластеров многочастичного распада нестабильного ядра. По своей идентификационной способности и информативности такой комплекс является уникальным в изучении структуры экзотических ядер, получаемых в реакциях с ультрамалыми выходами вблизи границ ядерной стабильности. Высокая гранулярность каждого детекторного модуля может быть эффективно использована в изучении угловых, энергетических и временны́х корреляций многочастичных распадов экзотических ядер.

Авторы выражают благодарности В.В. Авдейчикову и Е. А. Шевчику за плодотворную помощь в изготовлении сцинтилляционных детекторов и испытании их на пучках тяжелых ионов. 


\section{ЛИТЕРАТУРА}

1. Artukh A. G. et al. Wide aperture kinematic separator COMBAS realized on the strong focusing principle // Nucl. Instr. Meth. A. 1999. V.426. P. 605.

2. Артюх А. Г. и др. Фрагмент-сепаратор КОМБАС // ПТЭ. 2011. № 5. С. 74-87.

3. Fabris L., Madden N.W., Yaver H. A fast, compact solution for low noise charge preamplifiers // Nucl. Instr. Meth. A. 1999. V.424. P. 545-551.

4. Bertuccio G., Rehak P., Xi D. A novel charge sensitive preamplifier without the feedback resistor // Nucl. Instr. Meth. A. 1993. V. 326. P. 71-76.

5. Немчинов В.М. и др. Усилители с полевыми транзисторами. / Под ред. И.П. Степаненко. М.: Сов. радио, 1980.

6. Гусев В. Г., Гусев Ю. М. Электроника. М.: Высш. шк., 1991.

7. Гмурман В. Е. Теория вероятностей и математическая статистика. М.: Высш. шк., 2003.

Получено 17 марта 2014 г. 


\section{Редактор E. В. Сабаева}

Подписано в печать 14.04.2014.

Формат $60 \times 90 / 16$. Бумага офсетная. Печать офсетная.

Усл. печ. л. 1,1. Уч.-изд. л. 1,4. Тираж 245 экз. Заказ № 58239.

Издательский отдел Объединенного института ядерных исследований 141980 , г. Дубна, Московская обл., ул. Жолио-Кюри, 6.

E-mail: publish@jinr.ru www.jinr.ru/publish/ 